\title{
Resveratrol Oxidation in Botrytis cinerea Conidia
}

\author{
Marielle Adrian, Homa Rajaei, Philippe Jeandet, Jérôme Veneau, and Roger Bessis
}

First, fourth, and fifth authors: Laboratoire des Sciences de la Vigne, Institut Jules Guyot, Université de Bourgogne, BP 138, 21004 Dijon Cedex, France; second author: Biology Department, College of Sciences, Shiraz University, Shiraz, Iran; and third author: Laboratoire d'oenologie, UFR Sciences Exactes et Naturelles, Moulin de la Housse, BP 1039, 51687 Reims Cedex 2, France. Accepted for publication 6 February 1998.

\begin{abstract}
Adrian, M., Rajaei, H., Jeandet, P., Veneau, J., and Bessis, R. 1998. Resveratrol oxidation in Botrytis cinerea conidia. Phytopathology 88:472-476.

Observations using light microscopy showed that approximately $30 \%$ of Botrytis cinerea conidia treated with semi-lethal concentrations (i.e., $60 \mu \mathrm{g} / \mathrm{ml}$ ) of the grapevine phytoalexin resveratrol possessed intracellular brown coloration. This coloration was never observed in the absence of resveratrol or in conidia treated with resveratrol together with sulfur

dioxide (antioxidant compound) or sodium diethyldithiocarbamate (inhibitor of laccase action), suggesting that discoloration resulted from the laccase-mediated oxidation of resveratrol. Further studies using transmission electron microscopy enabled the observation of particular intravacuolar spherical vesicles and of granular material deposits along the tonoplast. These observations are likely to be related to the oxidation of resveratrol by an intracellular laccase-like stilbene oxidase of $B$. cinerea.
\end{abstract}

The production of phytoalexins is considered to be part of the general defense mechanism of grapevines $(6,13,17,27,28)$. In Vitis spp., such a response includes the formation of a range of biosynthetically related di- and oligomers of a simple stilbene, resveratrol (trans-3,5,4'-trihydroxystilbene) $(6,17,21,24,26,31)$, together with the formation of a dimethylated stilbene, pterostilbene (trans3,5-dimethoxy-4'-hydroxystilbene) (25). These compounds are typically accumulated in leaves and berries in response to stresses that include fungal infection, UV irradiation, and induction by chemicals $(1,5,24,26,31)$.

Resveratrol is quantitatively the major component in grapevine phytoalexin response. Its accumulation is correlated with resistance to various fungal organisms $(3,6,20,21,24,42)$ and, at physiological concentrations, is fungitoxic against Botrytis cinerea, the causal agent for gray mold (2). However, previous results have shown that $B$. cinerea secretes a laccase-like stilbene oxidase capable of oxidizing resveratrol $(16,22,40,41)$.

This work presents particular cytological and ultrastructural characteristics of $B$. cinerea conidia after exposure to resveratrol modified by laccase activity. These characteristics, which have never been described, are of particular relevance in the study of the $B$. cinerea-grapevine interaction.

\section{MATERIALS AND METHODS}

Culture of $B$. cinerea and preparation of conidial suspensions. The $B$. cinerea strain used for this work was collected at the viticultural and enological experiment station of the University of Bourgogne on mature grape berries of cultivar Pinot noir. Conidia were suspended in water, inoculated on potato dextrose agar (Bio Mérieux Corp., Lyon, France) and incubated at $21^{\circ} \mathrm{C}$. The strain was purified by several successive subcultures and the use of streptomycin (200 mg/liter). The single-spore strain was then stored at $4^{\circ} \mathrm{C}$ in tubes containing potato dextrose agar slants.

For further experimentation, the strain was grown on potato dextrose agar in $86-\mathrm{mm}$ petri dishes at $21^{\circ} \mathrm{C}$ with a 12 -h light photo-

Corresponding author: M. Adrian; E-mail address: sylvain.debord@u-bourgogne.fr

Publication no. P-1998-0325-02R

(C) 1998 The American Phytopathological Society period. Conidia were aseptically harvested from 10-day-old cultures by suction (39) and transferred into 250-ml Erlenmeyer flasks containing $100 \mathrm{ml}$ of a liquid nutrient-rich medium described by Fournioux and Bessis (11) and similar to that of Hoos and Blaich (16) or Ward and Stoessl (45). The conidial suspension was vibrated for 2 to 5 min with a vortex mixer and diluted to obtain a final concentration of about $10^{5}$ conidia per ml. The conidial suspension was then added to sterile $35-\mathrm{mm}$ petri dishes and immediately incubated with resveratrol.

Incubation of resveratrol with the conidial suspension. Prior to incubation, the purity of resveratrol was checked by high-pressure liquid chromatography (HPLC) analysis using a photodiode array detector $(1,19)$ and by gas chromatography (GC) as previously described (18). Resveratrol (Sigma Chemical Co., St. Louis) was added as an ethanolic solution to the nongerminated conidial suspension at $60 \mu \mathrm{g} / \mathrm{ml}$. The final concentration of ethanol in the culture medium was $4 \%$ to ensure solubility of the stilbene (41). Under these conditions, conidial germination and mycelial growth of $B$. cinerea were not inhibited (data not shown). The control contained just $4 \%$ absolute ethanol in the conidial suspension. Sodium diethyldithiocarbamate, $10 \mathrm{mM}$ (an inhibitor for laccase) $(23,29,30,36,46)$, or sulfur dioxide, $0.23 \mathrm{mM}$ (an antioxidative compound) (Prolabo, Paris) (7), was eventually added to the resveratrol solution to prevent its oxidation by B. cinerea laccase (29). Liquid cultures were protected from light (to avoid photochemical isomerization of the trans form of stilbenes to the less active cis form) (26), agitated with a reciprocal shaker $(120 \mathrm{rpm})$, and kept at $21^{\circ} \mathrm{C}$. There were five replicates per treatment and experiments were carried out 10 times.

Light microscopy. A drop of the conidial suspension was placed on a glass slide for each treatment and examined with a Leitz Laborlux D microscope (Leitz GmbH, Wetzlar, Germany). Staining with "neutral red" (Kuhlmann, Paris) was utilized to examine the vacuolar system.

Electron microscopy. After $48 \mathrm{~h}$ of incubation with an ethanolic solution of resveratrol or ethanol (control), B. cinerea suspensions were prepared for electron microscopy. Two samples were collected and examined per treatment (resveratrol and control). The suspensions of $B$. cinerea conidia were first passed through a Swinnex 13-mm filtration system (Poly Labo, Paul Block and Cie, Strasbourg, France) using a disk of Whatman paper (No. 1; Whatman 
International, Ltd., Maidstone, England) for filtration. Conidia were then retained on the filter paper. The following protocol was used to prepare conidial samples. The fungus was washed in $0.1 \mathrm{M}$ of phosphate buffer $(\mathrm{pH} 7.2)$ and added with fixing solution $(2.5 \%$ glutaraldehyde and $0.1 \mathrm{M} \mathrm{NaH} \mathrm{PO}_{4}-\mathrm{Na}_{2} \mathrm{HPO}_{4}, \mathrm{pH} 7.2$ ). After $4 \mathrm{~h}$ at $4^{\circ} \mathrm{C}$, samples were washed six times and left overnight in $0.1 \mathrm{M}$ of phosphate buffer $\left(0.1 \mathrm{M} \mathrm{NaH} \mathrm{PO}_{4}-\mathrm{Na}_{2} \mathrm{HPO}_{4}, \mathrm{pH}\right.$ 7.2) and then placed in citrate-phosphate buffer solutions of decreasing $\mathrm{pH}: 6.4$, 6.0, 5.4, and 5.0. Half of the samples (a control and one treated with resveratrol) were then dipped in a syringaldazine solution (3,5dimethoxy-4-hydroxy-benzaldehydazine; Sigma-Aldrich Chimie S.A.R.L., L'Isle d'Abeau, France; cytochemical reaction for laccase activity) in citrate buffer for 150 min followed by buffer replacement and overnight soaking. The other half of the samples were placed in the citrate buffer without syringaldazine. All samples were then placed in buffer solutions of increasing $\mathrm{pH}$ : 5.0, 5.4, 6.0, 6.4, and 7.2. The Swinnex filtration system was then dismounted and samples were postfixed in phosphate buffer, $\mathrm{pH} 7.2$, containing $1 \%$ (wt/vol) $\mathrm{OsO}_{4}$ for $4 \mathrm{~h}$ at $4{ }^{\circ} \mathrm{C}$. After careful washing in phosphate buffer and ultra-pure water (to avoid the precipitation of dimethoxypropane [DMP] by phosphate salts), dehydration was carried out in acidified DMP $(38,43)$ for $10 \mathrm{~min}$. DMP allows rapid dehydration and prevents the dissolution of the reaction product that occurs with acetone or absolute ethanol (15). The material was then removed from the filter and transferred to propylene oxide and embedded in Araldite-Epon resin mixture. Silver-gray sections were cut on a Sorvall MT-2B ultramicrotome (Ivan Sorvall, Inc., Newton, CT), mounted on copper grids, and stained in $2.5 \%$ uranyl acetate in $50 \%$ methanol for $30 \mathrm{~min}$, followed by lead citrate for 5 min. The sections were viewed in an $\mathrm{H}-600$ electron microscope (Hitachi Ltd., Tokyo) at an accelerating voltage of $75 \mathrm{kV}$ with a 30- $\mu \mathrm{m}$ objective aperture.

\section{RESULTS AND DISCUSSION}

Light microscopy observations of liquid cultures of $B$. cinerea conidia incubated with a sublethal concentration of resveratrol $(60$ $\mu \mathrm{g} / \mathrm{ml}$ ) showed that approximately $30 \%$ of the conidia contained an intracellular brown pigmentation after at least $48 \mathrm{~h}$ of incubation. This discoloration was either well delimited or occurred throughout
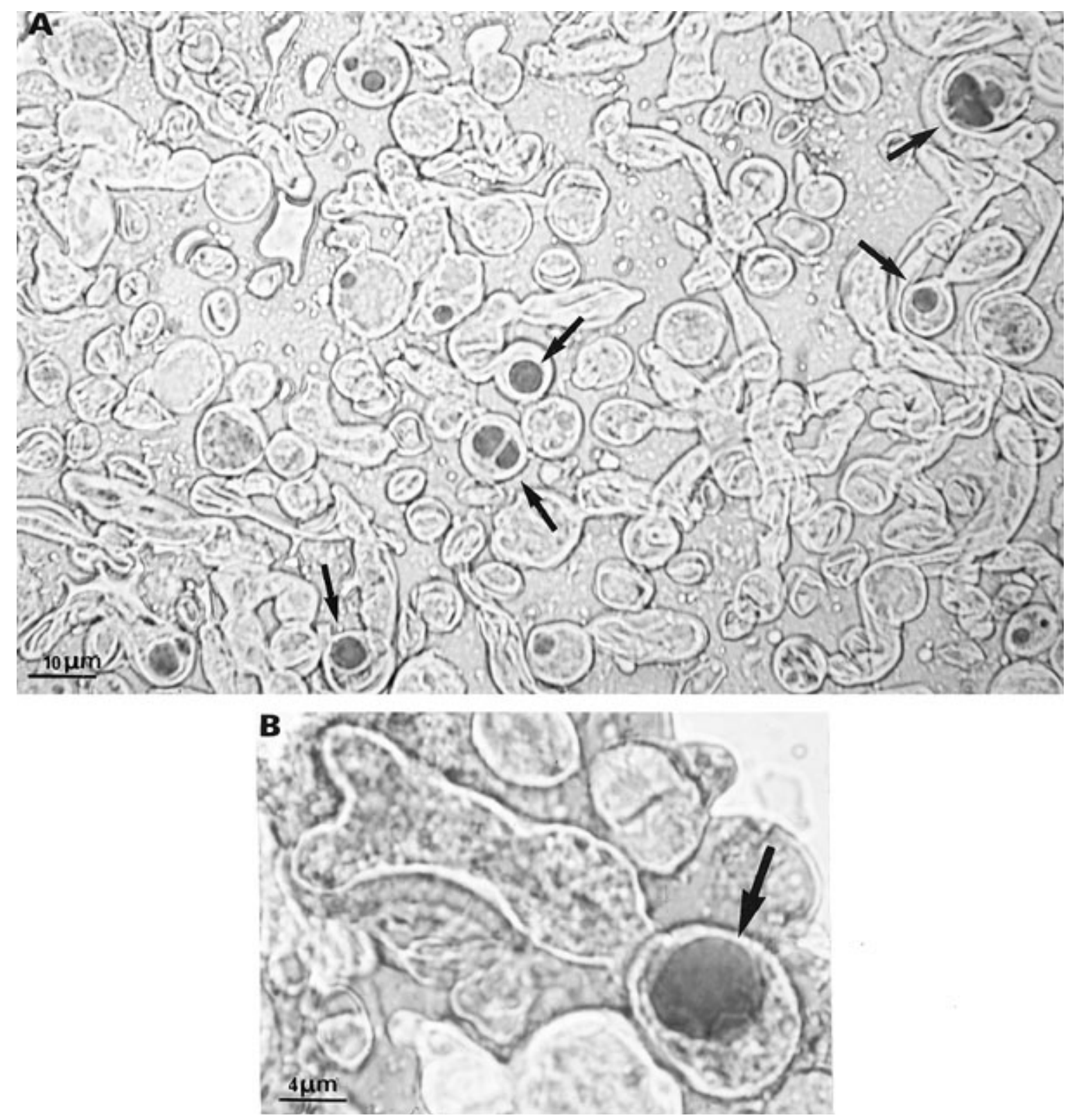

Fig. 1. Light microscopy observations of Botrytis cinerea conidia treated with $60 \mu \mathrm{g} / \mathrm{ml}$ of resveratrol for $48 \mathrm{~h}$. Note the occurrence of a brown coloration (arrows) present either $\mathbf{A}$, in the entire cytoplasm or $\mathbf{A}$ and $\mathbf{B}$, is clearly delimited. 
the entire conidial cytoplasm (Fig. 1). In contrast, no discoloration was evident in the controls. Brown coloration as described above has been reported in liquid cultures of $B$. cinerea containing hydroxystilbenes (16), but has never been described in fungal cell structures. According to these authors, this discoloration corresponds to an enzymatic oxidation of resveratrol by an extracellular polyphenol oxidase of $B$. cinerea (laccase-like enzyme) secreted in liquid cultures of this fungus. The discoloration observed within the conidia in our system could be attributed to a similar oxidation process. The fact that no discoloration was evident in the controls suggests that resveratrol, like many other phenolics, could act as an inducer of laccase production in B. cinerea conidia (32). In the model of Hoos and Blaich (16), disappearance of resveratrol from liquid culture media inoculated with $B$. cinerea was indeed accompanied by a brown pigmentation of the medium that could not be accelerated by the addition of $\mathrm{H}_{2} \mathrm{O}_{2}$. In our experiments, no coloration was observed in the culture medium containing conidia plus resveratrol, showing that $B$. cinerea conidia do not secrete laccase at this stage.

It is well established that $B$. cinerea produces an extracellular laccase-like polyphenol oxidase capable of oxidizing hydroxystil- benes, namely resveratrol $(16,22,40,41)$. Laccase ( $p$-diphenol:oxygen oxido-reductase, EC 1.10.3.2) (32,36), a glycoprotein produced either by plants $(23,29,35)$ or by fungi $(30)$, is capable of oxidizing a wide range of phenolics including mono-, di-, and triphenols $(34,36)$. An extracellular laccase from $B$. cinerea has been purified $(8,37)$. The molecular weight of $B$. cinerea laccase is approximately 60,000 (37), containing at least one copper atom per molecule, and shows unusual properties including a very low isoelectric point ( $\mathrm{pI}$ $2.5)(9,37,46)$. In the literature, the heterogeneity of the laccases from $B$. cinerea is clearly demonstrated; their properties mainly depend on the nature of their inducers (32). Our observations concerning the occurrence of a brown coloration in conidia (as a result of laccase-mediated oxidation of resveratrol) are in agreement with those of Mayer and Harel (36), describing the formation of highly colored products, often condensed molecules of high molecular weight, following the action of laccase on phenolic compounds.

We attempted to find out whether the discoloration observed in B. cinerea conidia could be attributable to laccase-mediated oxidation of resveratrol by incubating conidia plus resveratrol together with the antioxidative compound sulfur dioxide (7) or with
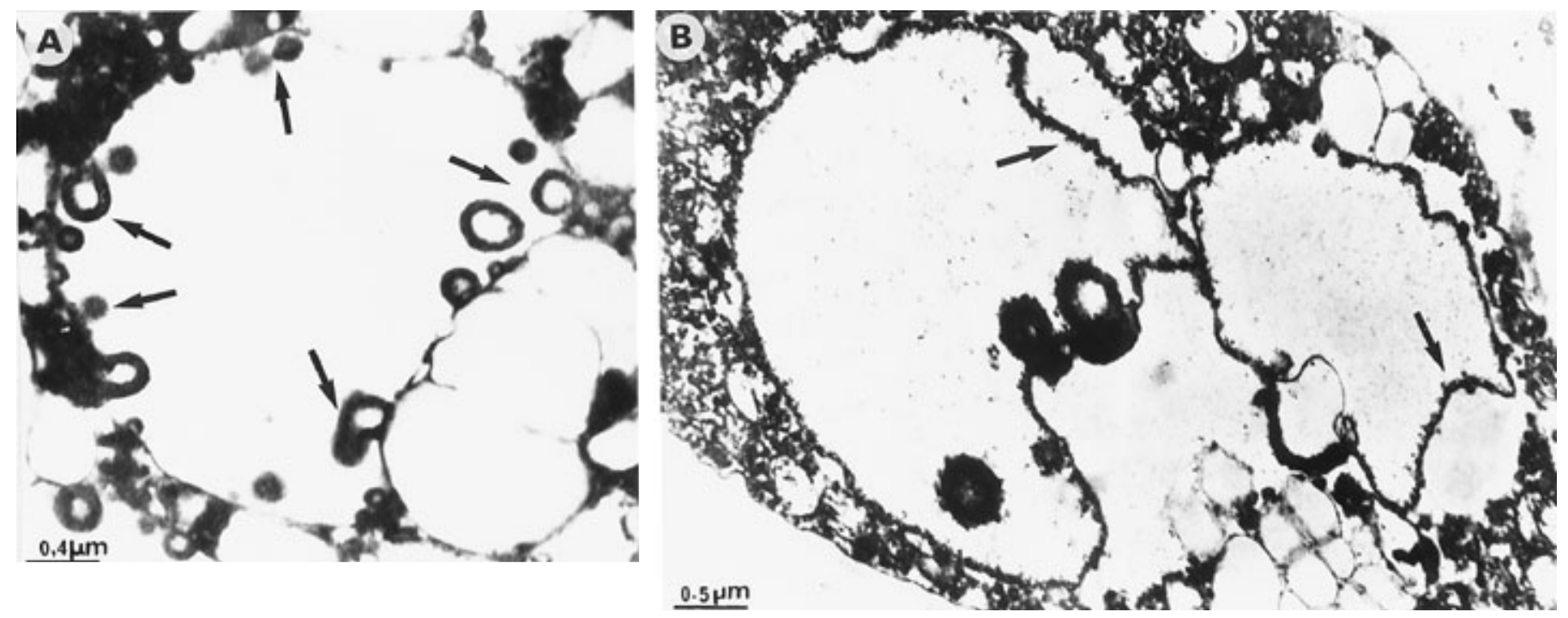

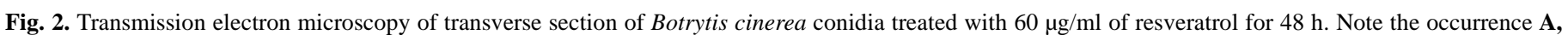
of vesicles (arrows) in the vacuoles and $\mathbf{B}$, of coarsely granular deposits (arrows) along the tonoplast.
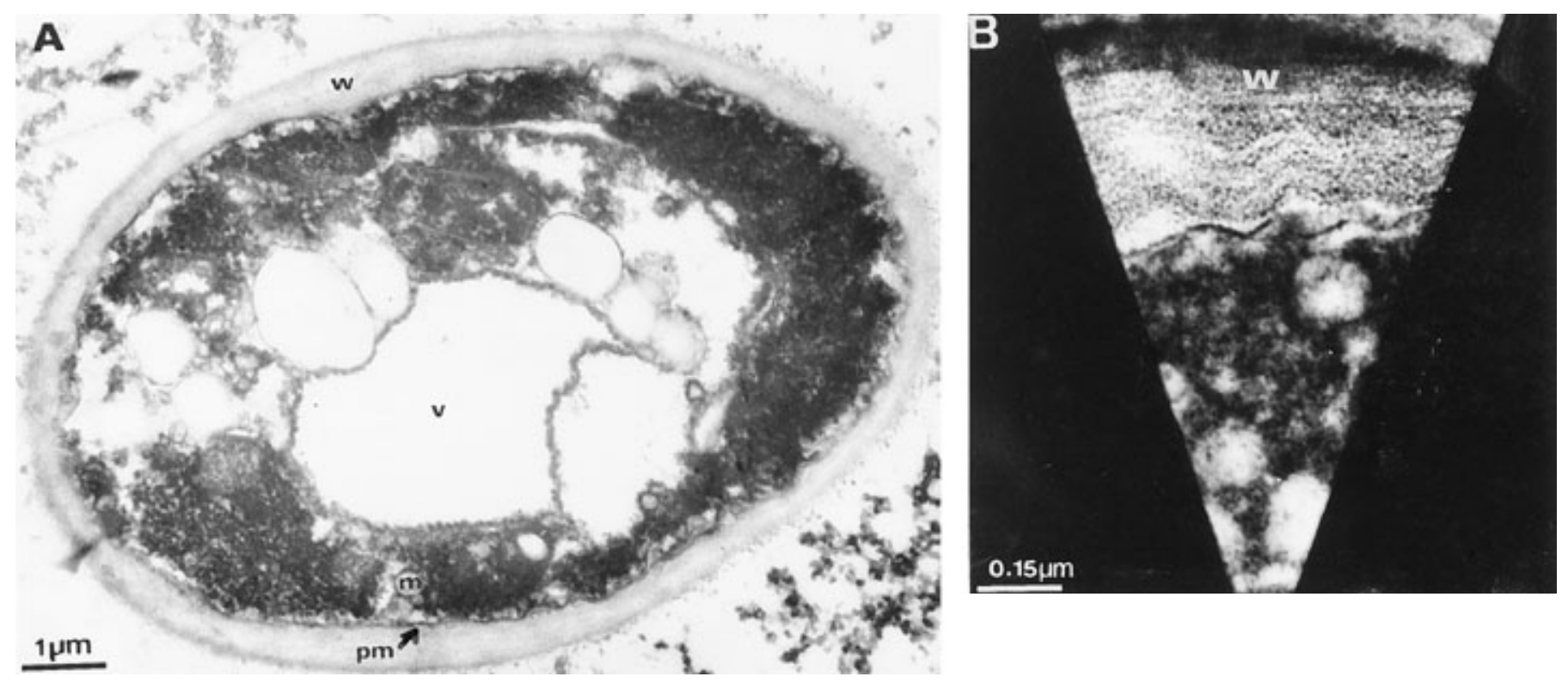

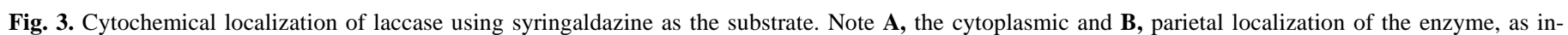
dicated by electron-dense deposits. Note that laccase is not detected in vacuoles. $\mathrm{m}=$ mitochondria, $\mathrm{pm}=\mathrm{plasma}$ membrane, $\mathrm{v}=\mathrm{vacuole}$, and $\mathrm{w}=\mathrm{wall}$. 
the specific laccase inhibitor sodium diethyldithiocarbamate (23, $29,30,36,46)$. In both cases, no discoloration was observed in conidia. These data thus suggest that an enzymatic process of resveratrol oxidation, specifically linked to a laccase-like polyphenoloxidase, takes place in the conidia of $B$. cinerea.

This oxidation process (or the accumulation of resveratrol oxidation products) could be located in vacuoles, because the area of the sporeling where the brown coloration was observed is also colored by neutral red, a staining specific for the vacuolar system.

An electron microscopy study showed that the ultrastructure of $B$. cinerea conidia treated with ethanol (control) was the same as that previously described by other authors $(4,12,39)$. However, some of the conidia treated with $60 \mu \mathrm{g}$ of resveratrol per ml presented different cytological abnormalities that have, to our knowledge, never been described. The tonoplast was often lined with electron-dense material, mainly along the vacuole (Fig. 2B). The brown discoloration observed in light microscopy could thus correspond to this coarse material present along the tonoplast. Spherical vesicles were also observed in the vacuole or along the vacuolar side of the tonoplast. They were either well delimited (by an electron-dense ring different from a membrane or a wall), fully or partly filled with coarsely granular material (in some cases), or even empty (Fig. 2A).

Vesicle size was also not uniform, varying from 0.1 to $0.4 \mu \mathrm{m}$. Different vesicular forms could be observed in the same vacuole. Their occurrence was restricted to conidia treated with resveratrol. They differ from the storage bodies described by Buckley et al. (4) and Gull and Trinci (12). The nature and function of these structures still remain unknown. Are they related to the oxidation process of resveratrol by fungal laccase, as they were not observed in the controls? If so, detoxification of resveratrol via the transport of oxidation metabolites into the vacuole is likely. The vacuole is often important in higher plants for detoxification of secondary metabolites. In plants, detoxification processes involve, at first, oxidation, reduction, or hydrolysis of the toxic product, followed by its conjugation to malonyl or glycosyl moieties by transferases and, finally, storage in the vacuole (33). Detoxification processes have also been described for fungi (44). The accumulation of resveratrol oxidation products in the vacuolar compartment of $B$. cinerea conidia could thus correspond to a detoxification process of this compound. Marbach et al. (32) strongly suggest that laccase is produced by $B$. cinerea conidia in order to deactivate plant defense mechanisms. The ability of $B$. cinerea conidia to metabolize resveratrol could thus contribute to creating a means of survival for conidia.

Since we observed the accumulation of resveratrol metabolites in the vacuole of $B$. cinerea conidia in relation to laccase activity, we wished to verify the localization of this enzyme in the fungal cell. Therefore, a cytochemical reaction using syringaldazine as a substrate for laccase $(10,14)$ was done. Syringaldazine produces unique electron-opaque deposits after oxidation that are visible by transmission electron microscopy (15). We observed the same electron-opaque deposits when syringaldazine was used as the substrate for laccase (Fig. 3). Past experiments indicate that laccase was located predominantly in the cytoplasm (15) and also in the cell wall and surrounding mucilages (which is expected, because laccase is an extracellular enzyme) (36). Neither laccase nor syringaldazine deposits were detected at the vacuolar level. Our experiments show that laccase activity is present within the conidial cell and its cytoplasm (not in vacuoles) (Fig. 3), and this is in agreement with the findings of other investigators. Thus, the laccasemediated oxidation of stilbenes takes place in the cytoplasm, and the resulting products later enter the vacuole where they accumulate, resulting in visible discoloration within the vacuole.

\section{ACKNOWLEDGMENTS}

We thank F. Marty, who enabled the realization of the transmission electron microscopy study at the Laboratoire de Phytobiologie Cellulaire, Université de Bourgogne, Dijon, France; D. Marty for her technical as- sistance; L. A. Weston at the University of Kentucky and L. M. Birden, ENSAM, Cluny, Burgundy, France, for reviewing the manuscript; and S. Debord and S. Kindt at the Laboratoire des Sciences de la Vigne, Université de Bourgogne, Dijon, France, and J. Relot at the Centre de Microscopie Appliquée á la Biologie, Dijon, France, for technical assistance.

\section{LITERATURE CITED}

1. Adrian, M., Jeandet, P., Bessis, R., and Joubert, J. M. 1996. Induction of phytoalexin (resveratrol) synthesis in grapevine leaves treated with aluminum chloride (AlCl3). J. Agric. Food Chem. 44:1979-1981.

2. Adrian, M., Jeandet, P., Veneau, J., Weston, L. A., and Bessis, R. 1997. Biological activity of resveratrol, a stilbenic compound from grapevines, against Botrytis cinerea, the causal agent for gray mold. J. Chem. Ecol. 23(7):1689-1702.

3. Barlass, M., Miller, R. M., and Douglas, T. J. 1987. Development of methods for screening grapevines for resistance to downy mildew. II. Resveratrol production. Am. J. Enol. Vitic. 38:65-68.

4. Buckley, P. M., Sjaholm, V. E., and Sommer, N. F. 1966. Electron microscopy of Botrytis cinerea conidia. J. Bacteriol. 91(5):2037-2044.

5. Creasy, L. L., and Coffee, M. 1988. Phytoalexin production potential of grape berries. J. Am. Soc. Hortic. Sci. 113:230-234.

6. Dercks, W., and Creasy, L. L. 1989. The significance of stilbene phytoalexins in the Plasmopara viticola-grapevine interaction. Physiol. Mol. Plant Pathol. 34:189-202.

7. Dubernet, M. 1974. Recherches sur la tyrosinase de Vitis vinifera et la laccase de Botrytis cinerea. Applications technologiques. Ph.D. thesis. University of Bordeaux II, France.

8. Dubernet, M., and Ribereau-Gayon, P. 1973. Les polyphénoloxydases du raisin sain et du raisin parasité par Botrytis cinerea. C. R. Acad. Sci. Paris 277(D):975.

9. Dubernet, M., Ribereau-Gayon, P., Lerner, H. R., Harel, E., and Mayer, A. 1977. Purification and properties of laccase from Botrytis cinerea. Phytochemistry 16:191-193.

10. Dubourdieu, D., Grassin, C., Deruche, C., and Ribereau-Gayon, P. 1984. Mise au point d'une mesure rapide de l'activit, laccase dans les moûts et dans les vins par la méthode à la syringaldazine. Application à l'appréciation de l'état sanitaire des vendanges. Conn. Vigne et Vin 18(4):237.

11. Fournioux, J. C., and Bessis, R. 1993. Use of carbon dioxide enrichment to obtain adult morphology of grapevine in vitro. Plant Cell Tissue Organ Cult. 33:51-57.

12. Gull, K., and Trinci, A. P. J. 1971. Fine structure of spore germination in Botrytis cinerea. J. Gen. Microbiol. 68:207-220.

13. Hain, R., Reif, H. J., Krause, E., Langebartels, R., Kindl, H., Vornam, B., Wiese, W., Schmelzer, E., Schreier, P., Stöcker, R., and Stenzel, K., 1993. Disease resistance results from foreign phytoalexin expression in a novel plant. Nature 361:153-156.

14. Harkin, J. M., and Obst, J. R. 1973. Syringaldazine, an effective reagent for detecting laccase and peroxydase in fungi. Experientia (Basel) 29:381-383

15. Hodson, M. J., Bar Nun, N., and Mayer, A. M. 1987. Cytochemical localization of laccase in Botrytis cinerea. Trans. Br. Mycol. Soc. 89:572-575.

16. Hoos, G., and Blaich, R. 1990. Influence of resveratrol on germination of conidia and mycelial growth of Botrytis cinerea and Phomopsis viticola. J. Phytopathol. 129:102-110.

17. Jeandet, P., Bessis, R., and Gautheron, B. 1991. The production of resveratrol $\left(3,5,4^{\prime}\right.$ trihydroxystilbene) by grape berries in different developmental stages. Am. J. Enol. Vitic. 42:41-46.

18. Jeandet, P., Bessis, R., Maume, B. F., Meunier, P., Peyron, D., and Trollat, P. 1995. Effect of enological practices on the resveratrol isomer content of wine. J. Agric. Food Chem. 43:316-319.

19. Jeandet, P., Breuil, A. C., Adrian, M., Weston, L. A., Debord, S., Meunier, P., Maume, B., and Bessis, R. 1997. HPLC analysis of grapevine phytoalexins coupling photodiode array detection and fluorometry. Anal. Chem. 69(24):5172-5177

20. Jeandet, P., Sbaghi, M., and Bessis, R. 1992. The use of phytoalexin induction and of in vitro methods as a tool for screening grapevines for resistance to Botrytis cinerea. Pages 109-112 in: Recent Advances in Botrytis Research. K. Verhoeff, N. E. Malathrakis, and B. Williamson, eds. Pudoc Scientific Publishers, Wageningen, the Netherlands.

21. Jeandet, P., Sbaghi, M., and Bessis, R. 1992. The production of resveratrol $\left(3,5,4^{\prime}\right.$-trihydroxystilbene) by grapevine in vitro cultures, and its application to screening for grey mould resistance. J. Wine Res. 3:47-57.

22. Jeandet, P., Sbaghi, M., and Bessis, R. 1993. The significance of stilbene-type phytoalexin degradation by culture filtrates of Botrytis cinerea in the vine-Botrytis interaction. Page 84 in: Mechanisms of Plant Defense Responses, B. Fritig and M. Legrand, eds. Kluwer Academic Publishers, Dordrecht, the Netherlands.

23. Joel, D., Marbach, I., and Mayer, A. M. 1978. Laccase in Anacardiaceae. Phytochemistry (Oxf.) 17:796-797. 
24. Langcake, P. 1981. Disease resistance of Vitis spp. and the production of the stress metabolites resveratrol, $\varepsilon$-viniferin, $\alpha$-viniferin and pterostilbene. Physiol. Plant Pathol. 18:213-226.

25. Langcake, P., Cornford, C. A., and Pryce, R. J. 1979. Identification of pterostilbene as a phytoalexin from Vitis vinifera leaves. Phytochemistry (Oxf.) 18:1025-1027.

26. Langcake, P., and Pryce, R. J. 1976. The production of resveratrol by Vitis vinifera and other members of the Vitaceae as a response to infection or injury. Physiol. Plant Pathol. 9:77-86.

27. Langcake, P., and Pryce, R. J. 1977. A new class of phytoalexins from grapevines. Experientia (Basel) 33:151-152.

28. Langcake, P., and Pryce, R. J. 1977. The production of resveratrol and the viniferins by grapevines in response to ultraviolet irradiations. Phytochemistry (Oxf.) 16:1193-1196.

29. Lehman, E., Harel, E., and Mayer, M. 1974. Copper content and other characteristics of purified peach laccase. Phytochemistry (Oxf.) 13:1713-1717.

30. Leoniwicz, A., and Malinowska, M. 1992. Purification of the constitutive and inducible forms of laccase of the fungus Pholiata mutabilis by affinity chromatography. Acta Biochim. Pol. 29(34):219-226.

31. Liswidowati, F., Melchior, F., Hohmann, F., Schwer, B., and Kindl, H. 1991. Induction of stilbene synthase by Botrytis cinerea in cultured grapevine cells. Planta 183:307-314.

32. Marbach, I., Harel, E., and Mayer, A. M. 1984. Molecular properties of extracellular Botrytis cinerea laccase. Phytochemistry (Oxf.) 23:2713-2717.

33. Martinoia, E., Grill, E., Tommasini, R., Kreuz, K., and Amrhein, N., 1993. ATP-dependant glutathione S-conjugate "export" pump in the vacuolar membrane of plant. Nature 364:247-249.

34. Mayer, A. M. 1987. Polyphenol oxidases in plants. Recent progress. Phytochemistry (Oxf.) 26(1):11-20.

35. Mayer, A. M., and Harel, E. 1968. A laccase-like enzyme in peaches. Phytochemistry (Oxf.) 7:1253-1256.

36. Mayer, A. M., and Harel, E. 1979. Polyphenol oxidases in plants. Phyto- chemistry (Oxf.) 18:193-215.

37. Mayer, A. M., Marbach, I., Marbach, A., and Sharon, A. 1977. Amino acid composition and molecular weight of Botrytis cinerea laccase. Phytochemistry (Oxf.) 16:1051-1052.

38. Müller, L. L., and Jacks, T. J. 1975. Rapid chemical dehydration of samples for electron microscopic examinations. J. Histochem. Cytochem. 23(2):107-110.

39. Pezet, R., and Pont, V. 1990. Ultrastructural observations of pterostilbene fungitoxicity in dormant conidia of Botrytis cinerea Pers. J. Phytopathol. 129:19-30.

40. Pezet, R., Pont, V., and Hoang-Van, K. 1991. Evidence for oxidative detoxication of pterostilbene and resveratrol by a lacase-like stilbene oxidase produced by Botrytis cinerea Pers. Physiol. Mol. Plant Pathol. 39:441-450.

41. Sbaghi, M., Jeandet, P., Bessis, R., and Leroux, P. 1996. Degradation of stilbene phytoalexins in relation to the pathogenicity of Botrytis cinerea to grapevines. Plant Pathol. 45:139-144.

42. Sbaghi, M., Jeandet, P., Faivre, B., Bessis, R., and Fournioux, J. C. 1995. Development of methods using phytoalexin (resveratrol) assessment as a selection criterion to screen grapevine in vitro cultures for resistance to grey mould (Botrytis cinerea). Euphytica 86:41-47.

43. Thorpe, J. R., and Harvey, D. M. R. 1979. Optimization and investigation of the use of 2,2-dimethoxypropane as a dehydration agent for plant tissues in transmission electron microscopy. J. Ultrastruct. Res. 68:186-194.

44. VanEtten, H. D., Matthews, D. E, and Matthews, P. S. 1989. Phytoalexin detoxification: Importance for pathogenicity and practical implications. Annu. Rev. Phytopathol. 27:143-164.

45. Ward, E. W. B., and Stoessl, A. 1972. Postinfectional inhibitors from plants. III. Detoxification of capsidiol, an antifungal compound from peppers. Phytopathology 62:1186-1187.

46. Zouari, N., Romette, J. L., and Thomas, D. 1987. Purification and properties of two laccase isoenzymes produced by Botrytis cinerea. Appl. Biochem. Biotechnol. 15:213-225. 